\title{
Neural evidence for the interplay between language, gesture, and action: A review
}

\author{
Roel M. Willems ${ }^{\text {a,* }}$, Peter Hagoort ${ }^{\text {a,b,c }}$ \\ ${ }^{a}$ F. C. Donders Centre for Cognitive Neuroimaging, Radboud University Nijmegen, P.O. Box 9101, 6500 HB Nijmegen, The Netherlands \\ ${ }^{\mathrm{b}}$ Max Planck Institute for Psycholinguistics, Wundtlaan 1, 6525 XD Nijmegen, The Netherlands \\ ${ }^{\mathrm{c}}$ Nijmegen Institute for Cognition and Information, Radboud University Nijmegen, P.O. Box 9104, 6500 HE Nijmegen, The Netherlands
}

Accepted 4 March 2007

Available online 9 April 2007

\begin{abstract}
Co-speech gestures embody a form of manual action that is tightly coupled to the language system. As such, the co-occurrence of speech and co-speech gestures is an excellent example of the interplay between language and action. There are, however, other ways in which language and action can be thought of as closely related. In this paper we will give an overview of studies in cognitive neuroscience that examine the neural underpinnings of links between language and action. Topics include neurocognitive studies of motor representations of speech sounds, action-related language, sign language and co-speech gestures. It will be concluded that there is strong evidence on the interaction between speech and gestures in the brain. This interaction however shares general properties with other domains in which there is interplay between language and action.
\end{abstract}

(C) 2007 Elsevier Inc. All rights reserved.

Keywords: Language; Action; Gesture; Cognitive neuroscience; Brain; ERP; Embodied cognition

\section{Introduction}

Co-speech gestures embody a form of manual action that is tightly coupled to the language system. Co-speech gestures and spoken language spontaneously occur together and both influence the understanding of a speaker's message (e.g. Goldin Meadow, 2003; Goldin Meadow, Kim, \& Singer, 1999; Goldin Meadow \& Momeni Sandhofer, 1999; McNeill, Cassell, \& McCullough, 1994; Singer \& Goldin Meadow, 2005). This strong coupling between gestures and speech has even led some to hypothesize that they belong to one integrated system of communication (e.g. McNeill, 1992). Recent years have seen the emergence of research in which the neural basis of co-speech gestures is studied. These studies will be reviewed below.

However, in order to evaluate the specificity of the relation between language and co-speech gesture, we feel that a

\footnotetext{
${ }^{*}$ Corresponding author. Fax: +31 243610989.

E-mail address: roel.willems@fcdonders.ru.nl (R.M. Willems).
}

broader perspective is needed. This perspective is that of the relation between language and action in general, of which the relation between language and gesture is a specific instantiation. We will first present our broader perspective and review relevant domains for our understanding of the relation between language and action. This provides the context for a discussion of studies on the neural basis of co-speech gestures.

The divide between cognition on the one hand and bodily action on the other has been central within cognitive science. Although several theorists have underlined the importance of action for cognition (e.g. Gibson, 1966; Merleau-Ponty, [1945]1962; Piaget \& Inhelder, [1966]1969), action and cognition are mostly studied as separate domains. This can be regarded as a reflection of traditional 'Cartesian' or 'orthodox' cognitive science. Mind and body are fundamentally different entities in this framework although they closely interact with each other. This strong metaphysical divide has led to the common notion that cognition is separated from action (see Clark, 1997; 
Noe \& Thompson, 2002; van Gelder, 1995; Varela, Thompson, \& Rosch, 1991; Wheeler, 2005 for discussion). Consequently, language - as a sub-domain of cognitionand action have also been traditionally studied as distinct faculties of the mind (Fodor, 1983). From an embodied cognition viewpoint however, contrary to the classical Cartesian view, the importance of bodily processes for cognition is central. Cognition is thought of as 'grounded' in and therefore inseparable of bodily action (see Anderson, 2003; Chiel \& Beer, 1997; Clark, 1997; Clark \& Chalmers, 1998; Noe \& Thompson, 2002; Pecher \& Zwaan, 2005; Varela et al., 1991; Wheeler, 2005; Wilson, 2002). The representation of a concept is for instance proposed to be crucially dependent upon perceptual-motor processes that relate to that concept (e.g. Gallese \& Lakoff, 2005). This stands in contrast to a more traditional, 'cognitivist' stance in which a concept's meaning is considered to be an amodal symbolic representation (e.g. Fodor, 1975). In its effort to unite cognition and action, embodied cognition comes in very different degrees, from only slightly modifying the traditional Cartesian view to radical claims about, for instance, the extension of the human mind into the environment (see Anderson, 2003; Chiel \& Beer, 1997; Clark, 1997; Clark \& Chalmers, 1998; Noe \& Thompson, 2002; Pecher \& Zwaan, 2005; van Gelder, 1995; Varela et al., 1991; Wheeler, 2005). These more and less radical proposals have in common that they stress the importance of connections between bodily actions and cognition or more specifically, action and language. This interrelatedness should ultimately be reflected in overlap of brain processes supporting language and action. Before discussing the relation between language and gesture, we will try to answer the more general question: What is the neural evidence for proposed connections between language and action? ${ }^{1}$

We have structured this review according to four different combinations of language and action that have been studied by cognitive neuroscience. First, at the most basic level are studies investigating the neural representations of speech sounds during comprehension. Some proposals argue that the neural representation of a speech sound during comprehension may involve structures in the motor cortex used in pronouncing a speech sound. This is a clear example in which researchers have looked for a neural link between language (speech sound) and action (motor cortex representation of the speech sound). Second, we review studies on the neural representation of action-related language. Here, the assertion is that the meaning of for instance an action verb is at least partially represented in the cortical motor system. In other words, to understand

\footnotetext{
${ }^{1}$ It is worth mentioning that there has been a growing interest in the interplay between language and action from a widely varying set of other fields of science in recent years, which will not be covered in this review. Examples are learning of concepts in artificial intelligence (Roy, 2005; Steels, 2003), developmental psychology (Bates \& Dick, 2002; Smith \& Thelen, 2003; Thelen \& Smith, 1994), and the evolution of language in evolutionary science (Arbib, 2005; Corballis, 2002).
}

action-related language, activations in the motor system are crucial. The idea of a linguistic entity (e.g. action word) as being represented in the neural motor system is comparable to that for speech sounds, however now at a higher, semantic level. A neural link between language and action would be shown in this case if indeed the perception of action verbs leads to activation of parts of the action network in the brain. Third, we look at studies on the neural correlates of sign language comprehension in deaf people. Sign languages are complete languages in which hand actions (signs) are used to communicate. Specifically, in this section we ask in what ways understanding sign language in deaf people is neurally different compared to understanding spoken language in hearing people. Overlap between neural processes of understanding sign language and spoken language would indicate that when linguistic information is fully conveyed through the hands, it taxes similar brain regions as when conveyed in spoken format. Lastly, we review studies on the neural basis of the comprehension of speech and co-occurring co-speech gestures. In these studies, language (speech) and action (co-speech gestures) crucially go together. Neural evidence for a link between language and action in this domain would be supported by the finding of overlapping brain processes for the comprehension of words and meaningful co-speech gestures.

Our review is selective in that we focus on studies using neuroimaging methods (including functional magnetic resonance imaging (fMRI), positron emission tomography (PET), event related potentials (ERPs) measured with electroencephalography (EEG), magnetoencephalography (MEG) and transcranial magnetic stimulation (TMS)). We have chosen not to include the extensive literature on lesion studies of action understanding, although we are aware of its relevance for the issues at stake. For reviews of the neuropsychological literature, we refer to a number of excellent papers (Saffran \& Scholl, 1999; Saygin, Wilson, Dronkers, \& Bates, 2004; Tranel, Kemmerer, Adolphs, Damasio, \& Damasio, 2003).

\section{Motor representations of speech sounds}

A first area in which the connection between language and action has been made is that of speech perception. Especially the motor theory of speech perception (Liberman \& Mattingly, 1985) has made strong claims about this connection. This theory posits that perceived speech sounds are directly mapped onto the motor representations of the articulatory gestures that would be necessary to produce these speech sounds. For example, upon hearing the word 'ball' the incoming sounds are mapped onto the stored motor representations that are recruited when pronouncing the word 'ball'. Crucially, in this theory, speech perception takes place by directly mapping the speech input onto a motor representation. In this way, the activation of motor representations is what constitutes speech perception.

In the light of the recent findings of mirror neurons in motor areas (di Pellegrino, Fadiga, Fogassi, Gallese, \& 
Rizzolatti, 1992; see Rizzolatti \& Craighero, 2004 for review) this theory has gained renewed popularity. The prediction that has been tested mostly is that perceiving a speech sound should activate the same motor representation in the brain as producing that speech sound. Note that this is a far less radical claim than proposed by the motor theory of speech perception. After all, activation of similar motor areas does not implicate that these are crucial for speech perception. We will get back to this after reviewing the studies on this topic.

Several neuroimaging studies have provided evidence for the hypothesis that perceiving a speech sound activates similar brain regions as producing that speech sound. In an fMRI experiment Wilson, Saygin, Sereno, and Iacoboni (2004) had subjects listen to meaningless syllables (e.g. $/ \mathrm{pa} /$ ) and subsequently pronounce the same syllables. They report overlapping activations during perception and production in bilateral precentral gyri and central sulci (premotor and primary motor cortex). Activation in these areas was significantly higher to the perception of the syllables than to non-speech sounds (e.g. the sound of a bell). In a related experiment, it was found that producing syllables which start with phonemes that involve movement of the lips or of the tongue ([p] and [t], respectively) activated the same portions of precentral cortex as listening to these syllables (Pulvermuller et al., 2006). Moreover, these regions overlapped with those activated when subjects simply moved their tongue or lips, that is, without speech production. Note that the cortical motor system at this level has a somatotopic organization, such that distinct parts command different effectors in a structured manner. Pulvermuller et al. showed that when listening to or pronouncing syllables related to lip or tongue movements, the same organization is preserved. In other words, parts of premotor cortex that become activated when moving the lips or tongue also become activated when listening to speech sounds that involve movement of the lips or tongue. In an interesting variant of this line of research, Wilson and Iacoboni (Wilson \& Iacoboni, 2006) pursued the issue of mapping speech sounds onto motoric representations by presenting English subjects with native (English) and non-native phonemes. The non-native phonemes were rated to be hard to pronounce by native speakers of English. The assumption of this study was that no motor representations would exist for the non-native phonemes, since they are not produced by speakers of English. Activity in superior temporal areas was negatively correlated with the producibility of a phoneme. That is, the harder a phoneme was to produce for the English speakers, the more superior temporal areas were activated. Premotor cortex also distinguished between native and non-native phonemes, in the sense that activation was higher for nonnative than for native phonemes. The authors argued that since a match between stored and perceived speech sounds is impossible, hearing a non-native speech sound led to an activation increase. These data neither support an exclusively sensory (reflected in superior temporal activation) nor motor (reflected in premotor activation) account of speech perception.

Overall, these fMRI studies suggest that merely listening to speech sounds activates parts of the neural motor system. Moreover, this activation seems to be rather specific in the sense that listening to speech sounds which involve movement of the lips also leads to activation of part of the motor cortex involved in actually moving the lips.

Another way of assessing whether motor cortex is activated during speech perception is to use TMS to measure the excitability of the motor cortex while subjects perceive speech. The method frequently used is to measure the motor evoked potential (MEP) in a muscle, while stimulating the motor cortex by a TMS pulse. The pulse will evoke an MEP when the subject is at rest. The rationale of this approach is that when a subject listens to speech, an increased excitability of the motor cortex will lead to an increased MEP. Note that the use of TMS described here is different from that in which a part of cortex is temporarily and virtually 'lesioned' (see Devlin \& Watkins, 2006).

Fadiga and colleagues measured MEPs of tongue muscles while stimulating the part of the motor cortex that leads to movement of the tongue (Fadiga, Craighero, Buccino, \& Rizzolatti, 2002). Subjects listened to Italian words containing phonemes that in production lead to strong movement of the tongue (e.g. 'birra') or to words that induce less tongue movement (e.g. 'baffo'). If listening to words evokes specific activation of the motor system, greater tongue muscle activation is expected to 'tongue words' than to 'non-tongue words'. Indeed, stronger MEPs were found when subjects listened to the 'tongue words' compared to when subjects listened to the 'non-tongue words'. Interestingly, this effect was also present with non-words with similar acoustic characteristics as the real words. However, in the non-words the MEP levels were smaller overall, which suggests a lexical influence on the motor representation of speech sounds.

Instead of looking at tongue muscles, Watkins, Strafella, and Paus (2003), measured the activity of lip muscles. They found increased MEPs of lip muscles in response to stimulation of the mouth representation in motor cortex while subjects listened to continuous prose. Hand muscles were recorded as a control condition while the cortical hand area was stimulated. No modulation of hand muscles was found. In contrast to this latter finding, Floel, Ellger, Breitenstein, and Knecht (2003) found facilitation of hand muscles to stimulation of hand motor cortex during speech perception. No such facilitation was found during a nonlinguistic memory task. This finding was explained in the context of an evolutionary scenario in which language is thought to have evolved out of manual communication. It is unclear why these authors do find modulation of hand muscles, whereas Watkins et al. (2003) did not. Watkins and Paus (2004) simultaneously measured activation across the whole brain using PET as well as MEPs of mouth muscles after stimulation with TMS. Activity in an area in the left inferior frontal cortex ('Broca's area') was correlated 
with the facilitation of the mouth muscle. The authors argue for Broca's area as 'priming' the motor system in response to speech perception.

Most TMS studies complement evidence from fMRI studies that found activation of parts of the motor cortex in listening to speech sounds (but see Sundara, Namasivayam, \& Chen, 2001). Facilitatory effects are reported for tongue, lip as well as hand muscles, depending upon the specifics of the experiment. The activation of motor cortex is reasonably fast, such that a TMS pulse given $100 \mathrm{~ms}$ after onset of a critical speech sound leads to a facilitation of the muscle involved in its production (Fadiga et al., 2002).

In sum, fMRI and TMS studies indicate that representations of speech sounds in (pre)motor cortex that are activated during speaking, are also activated while listening to speech sounds. Although it might be tempting to interpret this in terms of the motor theory of speech perception, note that the studies reviewed here do not provide unequivocal evidence that motor activation is necessary in understanding language, as was argued by the motor theory of speech perception (Galantucci, Fowler, \& Turvey, 2006). The finding of a lexical modulation of MEPs to words versus non-words with the same acoustic properties (Fadiga et al., 2002) for instance shows that speech sounds are not invariantly mapped onto motor representations, but are modulated by higher level processes (see also Wilson \& Iacoboni, 2006). Although these studies cannot prove the motor theory of speech perception, they do provide evidence for a link between language and action in the brain. After all, it is consistently reported that merely listening to speech or speech sounds activates part of the motor system involved in producing speech sounds. However, the status of these activations remains unclear.

\section{Action-related language}

So far, we have discussed a connection between language and action that is restricted to a form level without the requirement of access to the semantics of words. This is different for action-related language. The question here is: Do words describing actions activate parts of the brain involved in sensorimotor processes, such as premotor cortex? If so, this would provide evidence for a link between language and action in the brain because the assertion would be that parts of the brain that are activated when performing or observing an action are also activated when comprehending language describing that action.

Hauk, Johnsrude, and Pulvermuller (2004) took advantage of the somatotopic organization of the motor cortex to investigate the representation of action verbs. Subjects read verbs describing actions performed with the feet, hands or face (e.g. 'kick', 'pick', 'lick'). Subsequently, they performed simple actions with foot, finger or tongue, which activated primary and premotor cortex in a somatotopic fashion, as expected. Interestingly, reading action verbs led to a similar somatotopic pattern of activation. Overlap between parts of (pre)motor cortex activated by action verbs and by action production was clearly observed for two of the three effectors. Converging evidence for the involvement of motor areas in processing action words comes from a recent PET study, which reports increased activation in left precentral and central sulcus (i.e. premotor and primary motor cortex) when comparing motor words (both nouns and verbs) to sensory words (also both nouns and verbs) (Vigliocco et al., 2006).

Together, these studies provide evidence for the activation of premotor cortex in response to action words. Moreover, it shows that this activation is rather specific in the sense that action verbs performed with a certain effector elicit activation in the area that is also activated when using that effector.

A possible criticism is, however, that the activations in motor cortex may be the by-product of imagery of an action. This would mean that the premotor activations are not part of the representation of the action verb, but rather emerge only after the action concept is identified. Given the limited temporal resolution of the signal measured in fMRI and PET, this possibility cannot be excluded. Pulvermuller, Hauk, Nikulin, and Ilmoniemi (2005) argued against an explanation of their results in terms of mental imagery. To do so they looked at the timing of motor cortex activation to the action verbs. In a TMS study hand/arm and leg words were presented while stimulating the 'hand/arm' and 'leg area' of the motor cortex (Pulvermuller et al., 2005). Subjects' task was to make a lexical decision to the visually presented words. Faster reaction times were observed to hand/arm words after stimulation of the 'hand/arm area' as well as to leg words after stimulation of the 'leg area'. This was taken as evidence that the (pre)motor activation is a crucial part of the action verb's semantic representation, because stimulating the motor cortex speeded up lexical decision. In related MEG and EEG studies it was found that differences between leg, arm or face words emerge around $200 \mathrm{~ms}$ (Hauk \& Pulvermuller, 2004; Pulvermuller, Shtyrov, \& Ilmoniemi, 2005). Again, the localizations of these effects showed a dissociation between leg words in dorsal premotor cortex and arm and face words in more ventral premotor regions. The authors argue that the latency of this effect is too fast to be explainable in terms of general strategic effects such as imagery.

A related but partly different result than that of premotor cortex activation to action words was obtained in two fMRI studies (Kable, Kan, Wilson, Thompson-Schill, \& Chatterjee, 2005; Kable, Lease-Spellmeyer, \& Chatterjee, 2002). Neural activity in these studies was compared while subjects performed a semantic matching task on triads of pictures or words describing actions or objects. Subjects' task was to indicate which two objects or actions were semantically related to each other. In one of these studies increased activity to action words was observed in the motor cortex just as in the studies described above (Kable et al., 2005). However, additionally, increased activation 
was found in the vicinity of human motion area MT/MST (Kable et al., 2002, 2005), when subjects had to judge actions compared to when they had to judge objects. That is, the judging of actions evoked activity in areas implicated in the processing of motion which is implicated by actions but not by the observation of objects.

Two other studies in part confirmed the findings of activation of (pre)motor cortex to action words. Noppeney, Josephs, Kiebel, Friston, and Price (2005) report increased activation of left posterior temporal and anterior intraparietal areas in reaction to action words compared to abstract words. Only at a lower, uncorrected statistical threshold did they observe ventral premotor cortex activation. Ruschemeyer and Friederici (2006) compared activations to action verbs with 'complex verbs' that had the action verb as its stem, but did not have an action-related meaning. For example the German verb 'greifen' (to grasp) was compared to 'begreifen' (to understand). The rationale was that if action verbs automatically activate parts of the motor cortex, this should also be the case in complex verbs that include an action verb, such as 'begreifen'. Action verbs did activate premotor areas compared to abstract verbs. No such activation was however found in response to the complex verbs (i.e. verbs with an action stem but a non-action meaning). The conclusions to be drawn from this study however crucially depend on whether one believes that complex verbs such as 'begreifen' are stored with 'greifen' ('to grasp') as their stem.

An approach related to the studies of action words has been taken by a few studies comparing sentences describing actions to sentences describing abstract events. One fMRI study presented subjects with spoken sentences like 'I bite an apple' and 'I appreciate sincerity' (Tettamanti et al., 2005). The action sentences could be of three types: describing mouth actions ('I bite an apple'), hand actions ('I grasp the knife') or leg actions ('I kick the ball'). All action sentences activated left inferior frontal cortex more strongly than abstract sentences did. Moreover, sentences describing actions with different effectors activated the premotor cortex in a somatotopic manner. This result is comparable to that of action verbs which lead to premotor cortex activation in a somatotopic manner (Hauk et al., 2004). Aziz-Zadeh, Wilson, Rizzolatti, and Iacoboni (2006) found that action sentences activated premotor areas in a specific manner corresponding to whether sentences described an action done with the foot, hand or mouth. Moreover, the activation to a sentence type (i.e. foot, hand or mouth) was strongest in the area that was activated when the subject watched videos of that same type of action (actions performed with the foot, hand or mouth). Interestingly, this effect was not present when stimuli consisted of metaphors in which action verbs were used, such as 'He was chewing on the problem'.

Finally, a TMS study measured MEPs from hand and foot muscles while subjects listened to hand and foot related action sentences or to abstract sentences (Buccino et al., 2005). Stimulation of hand and foot areas in motor cortex led to decreased MEP amplitudes during perception of sentences related to the same effector; that is, for instance, stimulation of the hand area led to a decreased MEP in the hand muscle when subjects listened to a sentence implying an action done with the hand compared to a sentence describing an action done with the foot. Note that this is an opposite finding from other TMS studies with a similar design that generally report increases in MEP amplitudes, which is then interpreted as reflecting increased facilitation of the motor cortex. Buccino and colleagues related their opposite findings to the non-specificity of spoken language as compared to the observation of real, visual action. However, this does not explain the reversal of the effect compared to other studies using action words.

The results of a study by Boulenger et al. (2006) might shed light on the conflicting findings of Buccino et al. That is, Boulenger and colleagues measured arm movement kinematics of a grasping action that could either start slightly before the presentation of an action verb or after the presentation of an action verb. This manipulation resulted in a complete reversal of findings: when the word was processed before the onset of the action, grasping was facilitated. That is, the action was performed faster when preceded by an action verb than when preceded by a concrete noun (the control condition). However, when the action verb was read when the action had already started, the action was performed slower compared to the presentation of a concrete noun, indicating interference. As the authors note, this may explain why some TMS studies find an increase in MEPs (indicating facilitation), whereas others find a decrease in MEPs (indicating interference). Indeed, TMS pulses in Buccino et al. were delivered during the reading of an action verb, whereas they were applied shortly after the verb in other TMS studies (e.g. Pulvermuller et al., 2005). Future studies should systematically investigate the dynamics of information flow in these different cases.

Finally, a recent study used TMS to show that also abstract sentences invoke motor cortex activation (Glenberg et al., in press). Participants were presented with spoken sentences that described transfer (movement) of a concrete object, as in 'Andrea carries the pizza to you' or sentences describing the transfer of an abstract object, such as 'Arthur presents the argument to you'. It was hypothesized that the abstract sentences would be understood by activating the same transfer schema that is also used to comprehend literal transfer. Control sentences described events without the involvement of transfer (e.g. 'You smell the pizza with Andrea'). The main finding was an increase of MEPs to transfer sentences compared to non-transfer sentences. Moreover, there was no difference between concrete transfer sentences and abstract transfer sentences. This study suggests that not only concrete language describing an action activates part of the motor cortex, but that this also holds for abstract 'transfer sentences'.

In short, there is a considerable amount of evidence for the claim that listening to action-related language activates 
cortical motor areas. Whether motor cortex activation is a necessary part of the semantic representation of an action verb is not clear however. What the studies reviewed here show is that motor areas are activated upon hearing/reading an action word. To find out if the motor cortex is necessary in action word understanding, it needs to be shown that 'knocking out' the motor cortex (e.g. by repetitive TMS) specifically hampers the understanding of action words.

Another concern in most of these studies is the role of imagining what is being heard. Many results could reflect the contribution of motor imagery instead of the nature of the semantic representation. This alternative explanation cannot be easily discarded. In addition, it is not always easy to evaluate the weight of the conclusions, since most studies in this field are conducted without an explicit theory of semantic representation in mind. As a consequence, the precise implications of (pre)motor cortex activation are often unclear.

Despite these concerns, it is fair to conclude that the studies reviewed above provide ample evidence for a link between language and action, insofar as the activation of the motor cortex to action-related language is concerned.

\section{Sign language}

Sign language is the codified system in which hand signs are used to communicate with and by the deaf. Sign languages share many characteristics with spoken languages: signs are conventionalized, symbolic and produced in hierarchical combinations (Goldin Meadow, 2003; McNeill, 1992). They also clearly differ from spoken languages, since hand movements are used to communicate a message. An important question with regard to sign language is whether the comprehension of sign language requires similar neural activations as the comprehension of spoken language. A large degree of overlap would be evidence for a link between language and action, because it would show that hand actions lead to language-like processing when they are understood linguistically. Generally speaking, this is what most studies report. Many neuroimaging studies find the Perisylvian language network (comprising of left frontal and temporal areas) that is known to be activated when hearing participants process language, also activated when deaf signers watch American or British sign language (abbreviated ASL and BSL, respectively) (Levanen, Uutela, Salenius, \& Hari, 2001; MacSweeney et al., 2004, 2006; Newman, Bavelier, Corina, Jezzard, \& Neville, 2002, 1998, 2002). Although this suggests strong similarities between neural correlates of spoken and signed language, differences have been found as well, particularly with respect to the stronger involvement of parietal cortex (Emmorey et al., 2004, 2005; MacSweeney et al., 2002, 2004) as well as of the right hemisphere in signers (Neville et al., 1998; Newman et al., 2002).

The involvement of parietal areas in sign language is interesting since parietal areas have also been implicated in studies of action observation in hearing subjects. MacSweeney and colleagues (MacSweeney et al., 2004) directly compared activation in deaf signers to sentences of BSL and stretches of 'Tic Tac', a communication system used by bookmakers at horse races in Britain. None of the subjects were familiar with Tic Tac, whereas they clearly all were familiar with BSL. In this way MacSweeney et al. were able to compare the processing of linguistic hand actions (BSL) with non-linguistic hand actions that were produced with the intention to communicate (Tic Tac). Note that all participants in this study were aware of the fact that the Tic Tac and the BSL signs were made with the intention to be communicative. Stronger activations of bilateral inferior frontal, middle and superior temporal cortex, and, interestingly, the supramarginal gyrus were found to BSL compared to Tic Tac in deaf native signers. This study shows that inferior parietal (i.e. supramarginal gyrus) activation in users of sign language is a specific activation to signs and not to non-linguistic hand movements. Emmorey and colleagues were also interested in the action properties of sign language (Emmorey et al., 2004). They made use of the fact that signs in ASL of tools and actions performed with a tool, have a high degree of iconicity. That is, these signs resemble the action they represent to a considerable degree. In an fMRI study, production of these signs was compared to signs of actions with less iconicity. Production of all signs activated frontal and temporal language areas as well as parietal cortex compared to a low level baseline. No specific activation for iconic compared to non-iconic signs was observed however.

One thing to note is that in some studies the observation of sign language by hearing non-signers also leads to activation of traditional language areas, such as inferior frontal and/or superior temporal areas (Levanen et al., 2001; MacSweeney et al., 2004, 2006). Clearly, this cannot be due to the linguistic characteristics of the sign language, since the signs are meaningless to non-signers. There are two explanations for this finding. It is possible that observation of the hand actions activates part of the action recognition network, of which inferior frontal and superior temporal cortex are thought to be part (e.g. Rizzolatti \& Craighero, 2004; Rizzolatti, Fogassi, \& Gallese, 2001). It would mean that activations that in deaf signers can be attributed to the linguistic content of the stimuli, in nonsigners can be attributed to action observation. However, hearing naive subjects had instructions to assess the meaningfulness of the sign language expressions, even although they did not know any sign language (Levanen et al., 2001; MacSweeney et al., 2004, 2006). This mere 'looking for meaning' may provide an alternative explanation for the results that were obtained.

In sum, a language conveyed through the hands generally taxes similar parts of the brains of signers as spoken language does in the hearing. Interestingly, parts of the parietal cortex which are known to be involved in action understanding are activated more strongly in signers. This points to an interaction between linguistic and action 
processing which some have linked to an increased involvement of the mirror neuron system (Corina \& Knapp, 2006).

\section{Co-speech gestures}

Finally, we consider the link between language and action from the perspective of speech and accompanying co-speech gestures. Co-speech gestures are hand movements made during speaking. Some of these have a clear semantic relationship to the speech with which they are co-expressed. Consider for instance a person retelling a cartoon story while talking about 'the cat that rolled down the alley'. Together with the 'roll down' part of the utterance the speaker moves a finger in circles from one side to the other, as if the finger represents the character rolling down (e.g. Kita \& Özyürek, 2003; McNeill, 1992). Interestingly, these hand actions are almost never used without language. Consequently, they do not have a clear meaning outside of a language context, that is, people do not unambiguously recognize the intended meaning of co-speech gestures without speech (Krauss, Morrel Samuels, \& Colasante, 1991). Co-speech gestures are a core example of the interrelatedness of action and language since without language, they tend to lose their meaning.

Only recently neurocognitive studies have set out to investigate the neural underpinnings of co-speech gestures. Kelly, Kravitz, and Hopkins (2004) conducted an ERP study in which subjects saw an actor making a gesture corresponding to a property of an object, like its width or height. If the gesture had been preceded by a spoken word indicating a different property of the object, a stronger negative deflection was observed in the EEG signal compared to when word and gesture referred to the same property. This effect was maximal around $400 \mathrm{~ms}$ after the gesture and is commonly known as the N400 effect. In many language studies N400 effects are found when semantic processing of an item (i.e. a word) is harder to integrate into a previous context (see Brown, Hagoort, \& Kutas, 2000; Kutas \& van Petten, 1994, for review). Consequently, Kelly et al. argued for the N400 effect to index semantic processing as triggered by the hand gesture. In a follow-up study, Kelly, Ward, Creigh, and Bartolotti (2007) replicated the N400 effect to incongruent gestures. They furthermore showed that the effect size and its scalp distribution were modulated by whether subjects believed speech and gesture to be acted out by one person or not. That is, when subjects heard an utterance produced by one person while another person produced the accompanying hand gestures, N400 effect size and scalp distribution were different then when speech and gesture were coming from the same person. This was interpreted as reflecting the fact that semantic processing of gesture information is at least to some extent under cognitive control (see also Holle \& Gunter, in press). A related ERP study looked at the effect of presenting hand gestures after a more elaborate context, that is, an excerpt of a cartoon movie. Short movie clips of an actor performing a gesture that could either match the preceding cartoon or not, were shown. It was found that hand gestures that do not match a preceding cartoon movie also lead to an increased N400 (Wu \& Coulson, 2005). The evidence for gestures to evoke semantic processing was further supported by a study looking at the possibility that hand gestures could disambiguate the meaning of an otherwise ambiguous word (Holle \& Gunter, in press). Subjects listened to a sentence in which an ambiguous noun was accompanied by a gesture that hinted at the intended meaning of the ambiguous word. An N400 effect was observed to a word later in the sentence if the meaning of that later word did not match with the meaning indicated by the gesture earlier in the sentence.

These studies clearly indicate that co-speech gestures evoke semantic processing, a claim which had been debated in the literature before (Krauss et al., 1991; McNeill, 1992). However, a remaining question is how comparable the semantic processing evoked by hand gestures is to that of linguistic items such as words. This was investigated in a pair of studies measuring the neural time course (using ERPs) as well as the neural locus (using fMRI) of the sentence level integration of co-speech gestures and spoken words (Özyürek, Willems, Kita, \& Hagoort, in press; Willems, Özyürek, \& Hagoort, 2006). Subjects heard sentences in which a critical word was accompanied by a gesture. Either the word or the gesture could be semantically anomalous with respect to the context set up by the sentence. In the ERP study it was found that a semantically anomalous gesture as well as an anomalous word elicited an N400 effect. Importantly, the onset latencies of these effects did not differ. This implies that the time course of integration of meaningful information coming from gestures into a sentence context does not differ from the time course of integration of information conveyed through a spoken word. In the fMRI study, it was found that anomalous gesture as well as anomalous speech evoked increased activation in an overlapping region in left inferior frontal cortex. This region seems to play a crucial role in the integration of information coming both from speech and from gestures. Apart from overlapping activations, also effects specific to speech and gesture were found. Most interestingly, the gesture condition led to an increased activation of premotor cortex, an area activated by the observation of many types of actions. This study shows that besides being activated by the observation of an action, the activation level of premotor cortex is modulated by the semantic context of the action (gesture).

Another largely open question concerns how the presence or absence of co-speech gestures alters the neural response to speech. Skipper and colleagues (Skipper, Goldin Meadow, Nusbaum, \& Small, 2007) investigated this while measuring effective connectivity between brain areas. Effective connectivity is a measure of the influence one brain region exerts over the other during a given task. Note that this measure is related to the ongoing activation in the brain and not merely reflects the 'static' anatomical connections between areas. In their study, subjects watched a model tell a story. The model could produce natural hand 
gestures, produce 'self adaptor' movements (e.g. scratching herself, adjusting glasses) or leave the hands at rest. The results show that the speech and gesture condition led to a division of labor between areas related to language or action (Broca's area and premotor/primary motor cortex, respectively). That is, when speech was accompanied by natural gestures, Broca's area exerted the least influence on other areas. The authors explain this result as reflecting the decreased need for semantic selection or retrieval by Broca's area when gestures are present. It is claimed that the source of information provided by co-speech gestures helps the listener in understanding the speaker's message in such a way that less semantic control is needed. In other words, the presence of meaningful co-speech gestures as compared to no gestures or 'meaningless' self-adaptor movements allows the brain to use the additional source of information, decreasing the need for semantic control.

Another class of hand gestures relevant for this review are so-called emblems. Emblems are more or less conventionalized hand signs, such as 'thumbs up' meaning 'OK'. They are different from co-speech gestures in that emblems do have a meaning in the absence of speech. Two questions stand out here. First, how does neural activation in response to the combination of emblem and related word differ from neural activation to word or emblem alone? Second, how do neural indicators of semantic processing differ between emblems and words? As for the first question, Bernardis and Gentilucci (2006) studied articulation and arm movement characteristics of word and emblem production in varying combinations. Subjects either pronounced words, performed an emblem describing the word or both. The emblem and word combination led to a specific change in the acoustic properties (F2 formant) of the spoken word that was not present when the same word was produced alone or when a word was accompanied by a meaningless hand movement. Duration of hand movement decreased when speech and emblem conveyed the same meaning compared to producing only an emblem as well as compared to simultaneous production of a meaningless hand movement and meaningless word. Moreover, observation of speech and gesture combinations led to similar effects on the subsequent pronunciation of a word. In a follow-up study, similar stimuli were presented to subjects while inferior frontal cortex was disrupted by repetitive TMS (Gentilucci, Bernardis, Crisi, \& Dalla Volta, 2006). Disruption of left inferior frontal cortex led to a difference in acoustic characteristics of a word pronounced in reaction to a meaningful gesture compared to no TMS or TMS at right inferior frontal cortex. This effect was not observed when the word that was pronounced described a meaningless gesture. What these studies show is that production and comprehension of the combination of word and emblem leads to specific effects not present in production and comprehension of either word or emblem alone. Interestingly, this is true only when speech and action form a meaningful combination. Moreover, left inferior frontal cortex plays a crucial role in combining information from speech with information from an emblem.
The question how semantic processing evoked by an emblem might differ from that of a word, was investigated in two studies that measured EEG and MEG in reaction to the presentation of emblems versus meaningless hand shapes (Gunter \& Bach, 2004; Nakamura et al., 2004). It was found that meaningless emblems compared to meaningful emblems elicit increased N400 effects comparable to that observed for pseudo-words versus words (Gunter $\&$ Bach, 2004). In a MEG study, it was observed that right inferior parietal and right superior temporal cortices are more strongly activated to meaningful compared to meaningless emblems (Nakamura et al., 2004). This was interpreted as evidence for the involvement of areas known to be involved in action observation in coding the meaning of an emblem. Note that the signal measured in MEG allows for a much better localization of effects than the signal measured in EEG. From these two studies it seems as if the semantic processing evoked by meaningless versus meaningful emblems is similar as words versus pseudowords in terms of N400 effect. However, emblems seem to tax different brain regions as well, most notably areas involved in action observation.

Based upon the studies available it seems justified to conclude that processing evoked by gestures is qualitatively similar to that of words at the level of semantic processing. Note that although we have highlighted commonalities in neural activations to speech and co-speech gestures, interesting differences have also been observed. These differences indicate that the understanding of gestures accompanying speech is not the same as understanding spoken language. In other words, these gestures partly drive the neural language system, but also lead to specific effects not observed for spoken language. An example of this is the modulation of premotor areas when gestures are not in accordance with a previous sentence context (Willems et al., 2006).

By embedding speech and co-speech gestures research into other domains in which language and action have been studied together, we wanted to show that neural overlap between language and action is not only present in the case of speech and accompanying co-speech gestures. Rather, in this review it has become clear that language and action combinations exist at other levels of processing as well. Because of its nature, the combination of speech and cospeech gesture stands out however. Speech and gestures are crucially produced together and overlap at the level of semantics. This combination is arguably absent in the other domains of study that we have reviewed. Concretely, we hope that the overlap between these topics of investigation leads to useful new questions to be investigated in future studies. For instance, given the involvement of (pre)motor cortex in the observation of action words, it would be interesting to see how the dynamics of the motor system react to the observation of a meaningful gesture compared to a meaningless gesture. In the first case, the motor system would need to code both the kinematics as well as (perhaps) the meaning of the action. Another understudied 
issue concerns how the presence or absence of meaningful gestures affects the neural networks involved in understanding a message. The studies on emblems described above have convincingly shown that the combination of emblem and word leads to effects that are not simply a linear combination of perceiving either emblem or word alone (Bernardis \& Gentilucci, 2006; Gentilucci et al., 2006). One study has shown that the addition of a channel of meaningful information conveyed through the hand leads to a division of labor between cortical areas that is different from when speech is accompanied by meaningless gestures (Skipper et al., 2007). An important remaining question is how these effects are related to the degree of overlap between speech and meaningful gestures. That is, how do neural networks change when there is more or less information in meaningful gestures than in speech and vice versa?

\section{Discussion}

We have taken the co-occurrence of speech and cospeech gestures as a starting point for looking for neural links between language and action in general. In conclusion, studies in cognitive neuroscience provide evidence for links between language and action in the brain. First, motor areas activated in speech production are also activated when listening to speech sounds. Second, there is evidence for the involvement of the motor system in understanding action-related language. Third, purely manual languages (sign languages) recruit parts of cortex in deaf signers overlapping with those of spoken language in hearing subjects. Fourth, the understanding of meaningful co-speech gestures evokes similar neural processing as the understanding of words. In sum this can be taken as evidence in favor of an (embodied) approach to cognition in which language is thought to be grounded in bodily action. However, it is not the case that overlap between language and action systems is complete. First, in the literature on motor representations of speech sounds the question is how important motor cortex activation actually is in the understanding of speech. After all, the studies that we have reviewed do not provide decisive evidence for motor cortex to be necessary and/or sufficient for the understanding of speech. The same holds for activations of the motor system that are convincingly demonstrated in response to action-related language. Although these findings point to close neural links between language and action in the brain, the question is - again - whether motor cortex activation is necessary for understanding action-related language. Another word of caution in interpreting the overlap we have described between language and action systems concerns the studies on co-speech gestures and sign language. We have highlighted the overlap of processing co-speech gestures and signs with spoken language processing. Marked differences, however, also exist. Despite these cautionary notes, what is clear from this review is that language and action are not two isolated systems in the brain. Rather, language and action recruit overlapping parts of the brain and information coming from both domains can be used in qualitatively similar ways.

The more general question that needs to be addressed is how far the relation between language and action in the brain can be stretched. We want to argue against the kind of 'action-language chauvinism'2 in which understanding language is claimed to necessitate motor activation. Glenberg and Kaschak (2002) for instance write "... that language is made meaningful by cognitively simulating the actions implied by sentences" (p. 559). Similarly, Glenberg et al. (in press) write about "... an action schema in anterior portions of premotor cortex [...] which becomes the meaning of the verb "to give"”'(p. 8, our emphasis). As far as syntax is concerned, Glenberg (in press) hypothesizes that "...the mechanisms of action control provide the basis for combining ideas conveyed by language" (p. 22). In a similar vein, Gallese and Lakoff (2005) write: "The semantics of grammar is constituted by [...] structuring circuits used in the sensory motor system" (p. 473). In all these cases the assertion is that understanding language necessitates activating parts of the motor system. In contrast, we advocate a more balanced account of the neural basis of cognition as the dynamical interplay between several cognitive domains, without a particular emphasis on language and action. Large scale neural networks will be formed dynamically, involving those parts of the cortex that are needed by the specific task at hand for the organism (Fuster, 2003; Mesulam, 1990, 1998). In this way, the brain continuously crosses traditional boundaries of cognition when needed. To do this, a high degree of flexibility is needed. A good example of flexibility in the light of this review is the finding that action verbs when used metaphorically do not evoke somatotopic activation of the motor cortex as they do when used as literal action verbs (Aziz-Zadeh et al., 2006). In other words, activation of the premotor cortex in reaction to an action verb does not seem to be automatic, as was suggested by a radically embodied cognition stance (Gallese \& Lakoff, 2005). Rather, motor cortex activation can be overruled by the context the verb occurs in. This finding illustrates that the brain acts in a very flexible manner. This flexibility means that if the context renders the action interpretation of the verb (which is probably its default meaning) as incorrect, the motor cortex part of the verb's representation will not be activated.

Another characteristic of neural functioning that calls for a perspective that cuts across cognitive domains, is what we call the 'open-mindedness' of the brain. This implies that any type of information needed by the task at hand will be used. For instance, in the case of language comprehension, it has become increasingly clear that the brain uses several types of information in a qualitatively similar way to arrive at a full understanding of a message. This includes information from world-knowledge,

\footnotetext{
${ }^{2}$ This term is derived from Clark (2001, 2002), who accused proponents of a strong sensorimotor coupling (specifically O'Regan \& Noe, 2001) of 'sensorimotor chauvinism'.
} 
co-speech gestures, pictures, speaker's identity derived from voice characteristics and information from a preceding discourse (Federmeier \& Kutas, 2001; Hagoort, 2005; Hagoort, Hald, Bastiaansen, \& Petersson, 2004; Hagoort \& van Berkum, in press; Nieuwland \& van Berkum, 2006; Özyürek et al., in press; van Berkum, Hagoort, \& Brown, 1999; van Berkum, Zwitserlood, Hagoort, \& Brown, 2003; Willems et al., 2006). Importantly, these examples serve to demonstrate that the brain not only is capable of taking several streams of information into account, but actually does so in a qualitatively similar way.

The neural links between language and action that are described above are good examples of how the brain crosses the traditional boundaries of cognitive domains in a more flexible manner than traditionally thought. A possible implication of this which seems to be supported by the literature is that the same cortical region can be recruited for different cognitive processes. An example is Broca's area in left inferior frontal cortex. Traditionally this region was thought to subserve language production. Several studies indicate however that inferior frontal cortex is also recruited for other purposes, such as action observation, sequencing or semantic selection (e.g. Koechlin \& Jubault, 2006; Molnar-Szakacs, Iacoboni, Koski, \& Mazziotta, 2005; Thompson-Schill, D'Esposito, Aguirre, \& Farah, 1997). There are two ways to explain the multitude of conditions that make inferior frontal cortex 'light up'. First, one might try to classify all seemingly distinct tasks that activate this region under one common denominator. Thompson-Schill and colleagues, for instance, suggest that the inferior frontal cortex is involved in 'the regulation of mental activity' (Thompson-Schill, Bedny, \& Goldberg, 2005). Hierarchical processing is another suggested overarching function of the inferior frontal cortex (Koechlin \& Jubault, 2006; Tettamanti \& Weniger, 2006; see Friederici, 2006; van Schie, Toni, \& Bekkering, 2006 for related accounts). However, another way of conceiving the broad range of tasks activating inferior frontal cortex is to think of an area as a node in multiple different networks, in which the network and not the area instantiates a function (Fuster, 2003; Mesulam, 1990, 1998). That is, higher order cortex will be implicated in different functional networks. Note that this does not imply equipotentiality of cortical areas. It is clear that there is specialization in the brain, the degree of which might be different between areas, however (see Fuster, 2003; Mesulam, 1990, 1998). Neither of these views are mutually exclusive nor does strong evidence exist in favor of the one or the other. It does however seem that the latter view might prove to be more fruitful than searching for ever more abstract 'superfunctions' to be able to comply to the 'one area one function' rule.

In conclusion, the data reviewed here point to the fact that it is important and fruitful to study combinations between language and action. An increased awareness of the combination of language and action is warmly welcomed and supported by neuroscientific evidence to a considerable extent. This however should always be accompanied by an understanding of the brain as a flexible and open-minded system. Too strong an emphasis on the exclusive relation between language and action does not do justice to these characteristics.

\section{Acknowledgments}

This work was supported by Grant 051.02 .040 from the Dutch Organisation for Scientific Research (NWO). We thank Aslı Özyürek, Giosuè Baggio, Nina Davids, Doug Davidson, Floris de Lange as well as three anonymous reviewers for helpful comments on an earlier version of the manuscript.

\section{References}

Anderson, M. L. (2003). Embodied cognition: A field guide. Artificial Intelligence, 149, 91-130.

Arbib, M. A. (2005). From monkey-like action recognition to human language: An evolutionary framework for neurolinguistics. Behavioral and Brain Sciences, 28(2), 105-124.

Aziz-Zadeh, L., Wilson, S. M., Rizzolatti, G., \& Iacoboni, M. (2006). Congruent embodied representations for visually presented actions and linguistic phrases describing actions. Current Biology, 16(18), 1818-1823.

Bates, E., \& Dick, F. (2002). Language, gesture, and the developing brain. Developmental Psychobiology, 40(3), 293-310.

Bernardis, P., \& Gentilucci, M. (2006). Speech and gesture share the same communication system. Neuropsychologia, 44(2), 178-190.

Boulenger, V., Roy, A. C., Paulignan, Y., Deprez, V., Jeannerod, M., \& Nazir, T. A. (2006). Cross-talk between language processes and overt motor behavior in the first $200 \mathrm{msec}$ of processing. Journal of Cognitive Neuroscience, 18(10), 1607-1615.

Brown, C. M., Hagoort, P., \& Kutas, M. (2000). Postlexical integration processes in language comprehension: Evidence from brain-imaging research. In M. S. Gazzaniga (Ed.), The cognitive neurosciences (Second ed., pp. 881-895). Cambridge, Mass: MIT Press.

Buccino, G., Riggio, L., Melli, G., Binkofski, F., Gallese, V., \& Rizzolatti, G. (2005). Listening to action-related sentences modulates the activity of the motor system: A combined TMS and behavioral study. Brain Research Cognitive Brain Research, 24(3), 355-363.

Chiel, H. J., \& Beer, R. D. (1997). The brain has a body: Adaptive behavior emerges from interactions of nervous system, body and environment. Trends in Neurosciences, 20(12), 553-557.

Clark, A. (1997). Being there: Putting brain, body and world together again. Cambridge, MA: MIT press.

Clark, A. (2001). Visual experience and motor action: Are the bonds too tight? Philosophical Review, 110(4), 495-519.

Clark, A. (2002). Is seeing all it seems? Journal of Consciousness Studies, 9(5-6), 181-202.

Clark, A., \& Chalmers, D. (1998). The extended mind. Analysis, 58, 7-19.

Corballis, M. C. (2002). From hand to mouth: The origins of language. Princeton, NJ, US: Princeton University Press.

Corina, D. P., \& Knapp, H. (2006). Sign language processing and the mirror neuron system. Cortex, 42(4), 529-539.

Devlin, J. T., \& Watkins, K. E. (2006). Stimulating language: Insights from TMS. Brain, 130(3), 610-622.

di Pellegrino, G., Fadiga, L., Fogassi, L., Gallese, V., \& Rizzolatti, G. (1992). Understanding motor events: A neurophysiological study. Experimental Brain Research, 91(1), 176-180.

Emmorey, K., Grabowski, T., McCullough, S., Damasio, H., Ponto, L., Hichwa, R., et al. (2004). Motor-iconicity of sign language does not alter the neural systems underlying tool and action naming. Brain and Language, 89(1), 27-37. 
Emmorey, K., Grabowski, T., McCullough, S., Ponto, L. L., Hichwa, R. D., \& Damasio, H. (2005). The neural correlates of spatial language in English and American sign language: A PET study with hearing bilinguals. Neuroimage, 24(3), 832-840.

Fadiga, L., Craighero, L., Buccino, G., \& Rizzolatti, G. (2002). Speech listening specifically modulates the excitability of tongue muscles: A TMS study. European Journal of Neuroscience, 15(2), 399-402.

Federmeier, K. D., \& Kutas, M. (2001). Meaning and modality: Influences of context, semantic memory organization, and perceptual predictability on picture processing. Journal of Experimental Psychology: Learning, Memory, and Cognition, 27(1), 202-224.

Floel, A., Ellger, T., Breitenstein, C., \& Knecht, S. (2003). Language perception activates the hand motor cortex: Implications for motor theories of speech perception. European Journal of Neuroscience, 18(3), 704-708.

Fodor, J. A. (1975). The language of thought. Cambridge, MA: Harvard University Press.

Fodor, J. A. (1983). The modularity of mind. Cambridge, MA: MIT press.

Friederici, A. D. (2006). Broca's area and the ventral premotor cortex in language: Functional differentiation and specificity. Cortex, 42(4), 472-475.

Fuster, J. M. (2003). Cortex and mind. New York: Oxford University Press.

Galantucci, B., Fowler, C. A., \& Turvey, M. T. (2006). The motor theory of speech perception reviewed. Psychonomic Bulletin and Review, 13(3), 361-377.

Gallese, V., \& Lakoff, G. (2005). The brain's concepts: The role of the sensory-motor system in conceptual knowledge. Cognitive Neuropsychology, 22(3-4), 455-479.

Gentilucci, M., Bernardis, P., Crisi, G., \& Dalla Volta, R. (2006). Repetitive transcranial magnetic stimulation of Broca's area affects verbal responses to gesture observation. Journal of Cognitive Neuroscience, 18(7), 1059-1074.

Gibson, J. J. (1966). The senses considered as perceptual systems. Boston: Houghton Mifflin.

Glenberg, A.M. (in press). Language and action: Creating sensible combinations of ideas. In M.G. Gaskell (Ed.), The Oxford handbook of psycholinguistics. Oxford: Oxford University Press.

Glenberg, A. M., \& Kaschak, M. P. (2002). Grounding language in action. Psychonomic Bulletin and Review, 9(3), 558-565.

Glenberg, A.M., Sato, M., Cattaneo, L., Riggio, L., Palumbo, D., \& Buccino, G. (in press). Processing abstract language. Quarterly Journal of Experimental Psychology.

GoldinMeadow, S. (2003). Hearing gesture: How our hands help us think. Cambridge, MA, US: Belknap Press of Harvard University Press.

Goldin Meadow, S., Kim, S., \& Singer, M. (1999). What the teacher's hands tell the student's mind about math. Journal of Educational Psychology, 91(4), 720-730.

Goldin Meadow, S., \& Momeni Sandhofer, C. (1999). Gestures convey substantive information about a child's thoughts to ordinary listeners. Developmental Science, 2(1), 67-74.

Gunter, T. C., \& Bach, P. (2004). Communicating hands: ERPs elicited by meaningful symbolic hand postures. Neuroscience Letters, 372(1-2), $52-56$.

Hagoort, P. (2005). On Broca, brain, and binding: A new framework. Trends in Cognitive Sciences, 9(9), 416-423.

Hagoort, P., \& van Berkum, J.J. (in press). Beyond the sentence given. Philosophical Transactions of the Royal Society B.

Hagoort, P., Hald, L., Bastiaansen, M., \& Petersson, K. M. (2004). Integration of word meaning and world knowledge in language comprehension. Science, 304(5669), 438-441.

Hauk, O., Johnsrude, I., \& Pulvermuller, F. (2004). Somatotopic representation of action words in human motor and premotor cortex. Neuron, 41(2), 301-307.

Hauk, O., \& Pulvermuller, F. (2004). Neurophysiological distinction of action words in the fronto-central cortex. Human Brain Mapping, 21(3), 191-201.
Holle, H., \& Gunter, T.C. (in press). The role of iconic gestures in speech disambiguation: ERP evidence. Journal of Cognitive Neuroscience.

Kable, J. W., Kan, I. P., Wilson, A., Thompson-Schill, S. L., \& Chatterjee, A. (2005). Conceptual representations of action in the lateral temporal cortex. Journal of Cognitive Neuroscience, 17(12), 1855-1870.

Kable, J. W., Lease-Spellmeyer, J., \& Chatterjee, A. (2002). Neural substrates of action event knowledge. Journal of Cognitive Neuroscience, 14(5), 795-805.

Kelly, S. D., Kravitz, C., \& Hopkins, M. (2004). Neural correlates of bimodal speech and gesture comprehension. Brain and Language, 89(1), 253-260.

Kelly, S. D., Ward, S., Creigh, P., \& Bartolotti, J. (2007). An intentional stance modulates the integration of gesture and speech during comprehension. Brain and Language, 101, 222-233.

Kita, S., \& Özyürek, A. (2003). What does cross-linguistic variation in semantic coordination of speech and gesture reveal? Evidence for an interface representation of spatial thinking and speaking. Journal of Memory and Language, 48(1), 16-32.

Koechlin, E., \& Jubault, T. (2006). Broca's area and the hierarchical organization of human behavior. Neuron, 50(6), 963-974.

Krauss, R. M., Morrel Samuels, P., \& Colasante, C. (1991). Do conversational hand gestures communicate? Journal of Personality and Social Psychology, 61(5), 743-754.

Kutas, M., \& van Petten, C. K. (1994). Psycholinguistics electrified: Eventrelated brain potential investigations. In M. A. Gernsbacher (Ed.), Handbook of psycholinguistics (pp. 83-143). San Diego, CA: Academic Press.

Levanen, S., Uutela, K., Salenius, S., \& Hari, R. (2001). Cortical representation of sign language: Comparison of deaf signers and hearing non-signers. Cerebral Cortex, 11(6), 506-512.

Liberman, A. M., \& Mattingly, I. G. (1985). The motor theory of speech perception revised. Cognition, 21(1), 1-36.

MacSweeney, M., Campbell, R., Woll, B., Brammer, M. J., Giampietro, V., David, A. S., et al. (2006). Lexical and sentential processing in British sign language. Human Brain Mapping, 27(1), 63-76.

MacSweeney, M., Campbell, R., Woll, B., Giampietro, V., David, A. S., McGuire, P. K., et al. (2004). Dissociating linguistic and nonlinguistic gestural communication in the brain. Neuroimage, 22(4), 1605-1618.

MacSweeney, M., Woll, B., Campbell, R., Calvert, G. A., McGuire, P. K., David, A. S., et al. (2002). Neural correlates of British sign language comprehension: Spatial processing demands of topographic language. Journal of Cognitive Neuroscience, 14(7), 1064-1075.

MacSweeney, M., Woll, B., Campbell, R., McGuire, P. K., David, A. S., Williams, S. C., et al. (2002). Neural systems underlying British sign language and audio-visual English processing in native users. Brain, 125(7), 1583-1593.

McNeill, D. (1992). Hand and mind: What gestures reveal about thought. Chicago, IL, US: University of Chicago Press.

McNeill, D., Cassell, J., \& McCullough, K. E. (1994). Communicative effects of speech-mismatched gestures. Research on Language and Social Interaction, 27(3), 223-237.

Merleau-Ponty, M. ([1945]1962). Phenomenology of Perception. London: Routledge \& Kegan.

Mesulam, M. M. (1990). Large-scale neurocognitive networks and distributed processing for attention, language, and memory. Annals of Neurology, 28(5), 597-613.

Mesulam, M. M. (1998). From sensation to cognition. Brain, 121(6), 1013-1052.

Molnar-Szakacs, I., Iacoboni, M., Koski, L., \& Mazziotta, J. C. (2005). Functional segregation within pars opercularis of the inferior frontal gyrus: Evidence from fMRI studies of imitation and action observation. Cerebral Cortex, 15(7), 986-994.

Nakamura, A., Maess, B., Knosche, T. R., Gunter, T. C., Bach, P., \& Friederici, A. D. (2004). Cooperation of different neuronal systems during hand sign recognition. Neuroimage, 23(1), 25-34.

Neville, H. J., Bavelier, D., Corina, D., Rauschecker, J., Karni, A., Lalwani, A., et al. (1998). Cerebral organization for language in deaf 
and hearing subjects: Biological constraints and effects of experience. Proceedings of the National Academy of Sciences of the United States of America, 95(3), 922-929.

Newman, A. J., Bavelier, D., Corina, D., Jezzard, P., \& Neville, H. J. (2002). A critical period for right hemisphere recruitment in American Sign Language processing. Nature Neuroscience, 5(1), 76-80.

Nieuwland, M. S., \& van Berkum, J. J. A. (2006). When peanuts fall in love: $\mathrm{n} 400$ evidence for the power of discourse. Journal of Cognitive Neuroscience, 18(7), 1098-1111.

Noe, A., \& Thompson, E. (2002). Introduction. In A. Noe \& E. Thompson (Eds.), Vision and mind, selected readings in the philosophy of perception (pp. 1-14). Cambridge, MA: MIT press.

Noppeney, U., Josephs, O., Kiebel, S., Friston, K. J., \& Price, C. J. (2005). Action selectivity in parietal and temporal cortex. Brain Research Cognitive Brain Research, 25(3), 641-649.

O'Regan, J. K., \& Noe, A. (2001). A sensorimotor account of vision and visual consciousness. Behavioral and Brain Sciences, 24(5), 939-973.

Özyürek, A., Willems, R.M., Kita, S., \& Hagoort, P. (in press). On-line integration of semantic information from speech and gesture: Insights from event-related brain potentials. Journal of Cognitive Neuroscience.

Pecher, D., \& Zwaan, R. A. (Eds.). (2005). Grounding cognition: The role of perception and action in memory, language, and thinking. Cambridge, UK: Cambridge University Press.

Piaget, J., \& Inhelder, B. ([1966]1969). The psychology of the child. London: Routledge \& Kegan Paul.

Pulvermuller, F., Hauk, O., Nikulin, V. V., \& Ilmoniemi, R. J. (2005). Functional links between motor and language systems. European Journal of Neuroscience, 21(3), 793-797.

Pulvermuller, F., Huss, M., Kherif, F., Moscoso del Prado Martin, F., Hauk, O., \& Shtyrov, Y. (2006). Motor cortex maps articulatory features of speech sounds. Proceedings of the National Academy of Sciences of the United States of America, 103(20), 7865-7870.

Pulvermuller, F., Shtyrov, Y., \& Ilmoniemi, R. (2005). Brain signatures of meaning access in action word recognition. Journal of Cognitive Neuroscience, 17(6), 884-892.

Rizzolatti, G., \& Craighero, L. (2004). The mirror-neuron system. Annual Review of Neuroscience, 27, 169-192.

Rizzolatti, G., Fogassi, L., \& Gallese, V. (2001). Neurophysiological mechanisms underlying the understanding and imitation of action. Nature Reviews Neuroscience, 2(9), 661-670.

Roy, D. (2005). Grounding words in perception and action: Computational insights. Trends in Cognitive Sciences, 9(8), 389-396.

Ruschemeyer, S.-A., \& Friederici, A.D. (2006). Processing of motor vs. abstract words: An fMRI experiment. Paper presented at Architectures and Mechanisms for Language Processing (AMLaP), Nijmegen

Saffran, E., \& Scholl, A. (1999). Clues to the functional and neural architecture of word meaning. In C. M. Brown \& P. Hagoort (Eds.), The neurocognition of language. Oxford: Oxford University Press.

Saygin, A. P., Wilson, S. M., Dronkers, N. F., \& Bates, E. (2004). Action comprehension in aphasia: Linguistic and non-linguistic deficits and their lesion correlates. Neuropsychologia, 42(13), 1788-1804.

Singer, M. A., \& Goldin Meadow, S. (2005). Children learn when their teacher's gestures and speech differ. Psychological Science, 16(2), $85-89$.

Skipper, J. I., Goldin Meadow, S., Nusbaum, H. C., \& Small, S. L. (2007). Speech associated gestures, Broca's area and the human mirror system. Brain and Language, 101, 260-277.

Smith, L. B., \& Thelen, E. (2003). Development as a dynamic system. Trends in Cognitive Sciences, 7(8), 343-348.

Steels, L. (2003). Evolving grounded communication for robots. Trends in Cognitive Sciences, 7(7), 308-312.
Sundara, M., Namasivayam, A. K., \& Chen, R. (2001). Observationexecution matching system for speech: A magnetic stimulation study. Neuroreport, 12(7), 1341-1344.

Tettamanti, M., Buccino, G., Saccuman, M. C., Gallese, V., Danna, M., Scifo, P., et al. (2005). Listening to action-related sentences activates fronto-parietal motor circuits. Journal of Cognitive Neuroscience, 17(2), 273-281.

Tettamanti, M., \& Weniger, D. (2006). Broca's area: A supramodal hierarchical processor? Cortex, 42(4), 491-494.

Thelen, E., \& Smith, L. B. (1994). A dynamic systems approach to the development of cognition and action. Cambridge, MA: MIT Press.

Thompson-Schill, S. L., Bedny, M., \& Goldberg, R. F. (2005). The frontal lobes and the regulation of mental activity. Current Opinion in Neurobiology, 15(2), 219-224.

Thompson-Schill, S. L., D'Esposito, M., Aguirre, G. K., \& Farah, M. J. (1997). Role of left inferior prefrontal cortex in retrieval of semantic knowledge: A reevaluation. Proceedings of the National Academy of Sciences of the United States of America, 94(26), 14792-14797.

Tranel, D., Kemmerer, D., Adolphs, R., Damasio, H., \& Damasio, A. R. (2003). Neural correlates of conceptual knowledge for actions. Cognitive Neuropsychology, 20(3-6), 409-432.

van Berkum, J. J. A., Hagoort, P., \& Brown, C. M. (1999). Semantic integration in sentences and discourse: Evidence from the N400. Journal of Cognitive Neuroscience, 11(6), 657-671.

van Berkum, J. J. A., Zwitserlood, P., Hagoort, P., \& Brown, C. M. (2003). When and how do listeners relate a sentence to the wider discourse? Evidence from the N400 effect. Cognitive Brain Research, 17(3), 701-718.

van Gelder, T. (1995). The distinction between mind and cognition. In Y.H. Houng \& J.-C. Ho (Eds.), Mind and cognition. Taipei, Taiwan: Academia Sinica.

van Schie, H. T., Toni, I., \& Bekkering, H. (2006). Comparable mechanisms for action and language: Neural systems behind intentions, goals, and means. Cortex, 42(4), 495-498.

Varela, F. J., Thompson, E., \& Rosch, E. (1991). The embodied mind. Cambridge, MA: MIT Press.

Vigliocco, G., Warren, J., Siri, S., Arciuli, J., Scott, S., \& Wise, R. (2006). The role of semantics and grammatical class in the neural representation of words. Cerebral Cortex, 16(12), 1790-1796.

Watkins, K. E., \& Paus, T. (2004). Modulation of motor excitability during speech perception: The role of Broca's area. Journal of Cognitive Neuroscience, 16(6), 978-987.

Watkins, K. E., Strafella, A. P., \& Paus, T. (2003). Seeing and hearing speech excites the motor system involved in speech production. Neuropsychologia, 41(8), 989-994.

Wheeler, M. (2005). Reconstructing the cognitive world. Cambridge, MA: MIT press.

Willems, R. M., Özyürek, A., \& Hagoort, P. (2006). When language meets action: The neural integration of gesture and speech. Cerebral Cortex.

Wilson, M. (2002). Six views of embodied cognition. Psychonomic Bulletin and Review, 9(4), 625-636.

Wilson, S. M., \& Iacoboni, M. (2006). Neural responses to nonnative phonemes varying in producibility: Evidence for the sensorimotor nature of speech perception. Neuroimage, 33(1), 316-325.

Wilson, S. M., Saygin, A. P., Sereno, M. I., \& Iacoboni, M. (2004). Listening to speech activates motor areas involved in speech production. Nature Neuroscience, 7(7), 701-702.

Wu, Y. C., \& Coulson, S. (2005). Meaningful gestures: Electrophysiological indices of iconic gesture comprehension. Psychophysiology, 42(6), 654-667. 